\title{
Orthopaedic Practices and Surgeries during COVID-19 in Pakistan - A Survey Based Study
}

\author{
Saad-Ilyas M', FCPS, Zehra U², PhD, Khan UU3 , FCPS, Mohammad I', FCPS, Muhammad R5, MBBS, \\ Aziz A', FRCS \\ 'Department of Orthopaedics \& Spine Surgery, Ghurki Trust Teaching Hospital, Lahore, Pakistan \\ ${ }^{2}$ Department of Anatomy, University of Health Sciences, Lahore, Pakistan \\ ${ }^{3}$ Department of Orthopaedic, Kabir Medical College, Peshawar, Pakistan \\ ${ }^{4}$ Trauma Centre, Makhdoom Aali, Tehsil Dunyapur, Pakistan \\ ${ }^{5}$ Department of Orthopaedics, Chandka Medical College, Larkana, Pakistan
}

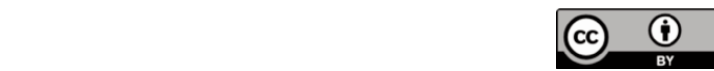

This is an open-access article distributed under the terms of the Creative Commons Attribution License, which permits unrestricted use, distribution, and reproduction in any medium, provided the original work is properly cited
\end{abstract}

Date of submission: 26th August 2020

Date of acceptance: 01 st February 2021

\begin{abstract}
Introduction: The study aimed to target the current practices of the orthopaedic community in outpatient (OPD), emergency (ER) and surgical services (OT) during COVID19.

Material and method: This study surveyed 303 orthopaedic surgeons from all over Pakistan. The survey had 30 questions targeting the setup of outpatient, emergency and operation services in orthopaedic departments of different hospitals in Pakistan.

Result: A total of 302 surgeons were included from 53 cities all over Pakistan. Between $35-48 \%$ of the respondents reported lack of availability of standard operating procedures in OPD, ER and in OT. Majority of the respondents noted that their OPD and surgical practice had been affected to some degree and $69 \%$ of the surgeons were only doing trauma surgery. This trend was higher in younger consultants of less than 45 years of age $(\mathrm{p}<0.001)$. Almost two-third of the surgeons, mostly senior $(\mathrm{p}=0.03)$ were using surgical masks as the only protective measure during various practices of OPD, ER and OT, while most of the setups were not assessing patients even for signs and symptoms of COVID. Almost $89 \%$ of the orthopaedic community is facing definite to mild stress during this pandemic and this has significantly affected the senior surgeons $(\mathrm{p}=0.01)$.

Conclusion: Our study highlighted that COVID-19 has resulted in marked changes to the practices of the majority of Pakistani orthopaedic surgeons. Despite a sharp upsurge in the number of cases and mortality due to COVID-19, guidelines were still lacking at most of the settings and a substantial percentage of the orthopaedic community were not following adequate safety measures while attending to patients.
\end{abstract}

Keywords:

COVID-19, coronavirus, orthopaedics, safety measures, pandemic

\section{INTRODUCTION}

The recent pandemic of Coronavirus disease (COVID-19) has affected the lives of people worldwide. However, the greatest burden has been placed on the medical profession. It has posed a huge challenge to the medical units and teams working around the world ${ }^{1,2}$. While the front line health care workers are facing daunting tasks of handling COVID-19 patients, the other medical sub-specialties are struggling hard to manage and maintain their essential services and indispensable workflow ${ }^{3}$. The main aim is to minimise the effect of the COVID-19 pandemic on other patient care areas. As a part of the large health-care network, orthopaedic surgeons also have a critical role to play during this pandemic. They are at risk from a variety of sources in both inpatient and outpatient settings as they have close patient contact during the management of both non-operative and operative orthopaedic cases ${ }^{4}$. The findings of a recently published study endorsed this notion and reported that $78 \%$ infected healthcare workers were non-front liners 5 . Hence, proper measures and guidelines must be practiced to halt or at least minimise the spread of infection as much as possible while attending to the patients.

In the face of the current pandemic, the heath care facilities of a developing country like Pakistan are getting stretched thin. Medical resources like the number of doctors and nursing staff, particularly the specialists, the equipment and protective-gear all in combine give an alarmingly fragile picture $^{6}$. Latest data shows a sharp upsurge of virus cases 
among health care workers and many deaths have been reported so far which is alarming ${ }^{7}$. Therefore, the careful prioritisation of trauma and orthopaedic surgical services must be planned keeping in view the clinical urgency, resource availability and protection of patient and healthcare workers 8 . The orthopaedic community in their own capacity is maintaining the work flow in different hospitals inclining to patients which require urgent or early orthopaedic care such as with musculoskeletal trauma and tumours ${ }^{9}$. In general the proper guidelines and safety measures must be taken into account to reduce the risk of infection in patients and medical personnel at different setups ${ }^{10}$, but as the case numbers are escalating further utmost care and caution is desirable.

In the current scenario, orthopaedic surgeons need to be aware of the hazards to patients and health care personnel in view of underdiagnosed cases. The understanding and protocols should be clear for pre-operative COVID-19 evaluation for all surgical cases ${ }^{11}$. Our local orthopaedic community in Pakistan varies widely with surgeons of extreme ages, expertise and resources, therefore, it is highly mandatory to assess and evaluate the current practices of orthopaedics in this COVID era for better understanding of existing health and safety measures. The current study has designed an anonymous online survey for orthopaedic surgeons of the country to gain valuable information regarding their setup during COVID-19 and to assess the practices they are following. This will help us to understand how the emergence of COVID-19 has impacted orthopaedic surgeons and the findings will lead us to guide policy makers to address the deficiencies and meet the current and future needs of our healthcare system.

\section{MATERIAL AND METHOD}

A survey consisting of 7 demographics, 18 other questions targeting outpatient (OPD), emergency (ER) and surgical practices, and 5 general questions (Table I) to understand the behaviour and state of mind of orthopaedic surgeons during this COVID duration was designed on Google forms survey. The survey had a total of 30 questions with 2 to 6 options each and was based on detailed discussion and agreement by the working group of 2 orthopaedic surgeons and 1 researcher. The survey link was sent via Emails, WhatsApp and Facebook to various orthopaedic surgeons in Pakistan using a non-probability snowball sampling technique. The survey was collected from 17th May to 5th June 2020 and reminders were sent on these platforms at various time points to participants to complete the survey. All categorical variables were presented as percentages. Data was entered in SPSS version 20 [IBM Corp] for statistical analysis. Chi square test was applied to see the statistical significance of age group on other categorical variables. Spearman rank correlation was used to evaluate relationships involving ordinal variables. Multiple logistic regression analysis was done to consider the simultaneous influence of several variables on the response. P-value $<0.05$ was considered significant.

\section{RESULTS}

The responses were collected from 53 cities all over Pakistan (Fig 1). In total, 303 individuals undertook the survey; one form that was completed by a foreign orthopaedic surgeon was excluded since the scope of the survey was to highlight the practices among Pakistani orthopaedic surgeons. Among the 302 respondents, 99\% were males, almost $63 \%$ were between the ages of $25-34,67 \%$ had completed their postgraduate training whereas the rest were residents and trainees. The demographic and work practice details are summarised in Table II.

In response to the query regarding standard operating procedures (SOP) laid down by the department during the COVID-19 pandemic in OPD, ER and operation theatres (OT), almost $35 \%$ of the respondents mentioned lack of availability of SOP in their OPD, 40\% stated this deficiency in ER and almost $48 \%$ in OT (Fig 2). Concerning the routine of the OPD, $75 \%$ of the doctors stated that they had changed their routine of OPD practice as compared to the pre-COVID era. Of these $30 \%$ had reduced the days, reduction in the staff had been done by $30 \%$ while $11 \%$ had reduced the timings. Private OPD practice had also been reported to be changed by almost $90 \%$ of the orthopaedic surgeons and almost $43 \%$ had stopped attending OPD while $40 \%$ were seeing only patients who expressed their urgent need.

Regarding the safety practices in OPD, only $5 \%$ opted for full PPE suit while 68\% were using surgical masks. Among them only $1 \%$ had combined it with a face shield and $1 \%$ were alternating it with an N95 respirator. However, only $27 \%$ were using N95 masks strictly in OPD, which drastically reduced to $16 \%$ in ER and $17 \%$ in OT. Majority of the respondents, that is $66 \%$ in ER and $75 \%$ in OT were using surgical masks only. In emergency services full PPE safety suit was only used by $15 \%$ of the individuals (Fig. 3). Interestingly the safety practices by the individuals were seen to be correlating in all the three areas of practice $(p<0.001)$ (Table III).

Only $66 \%$ of the orthopaedic emergency services were screening patients for COVID-19 with signs and symptoms on arrival, while $25 \%$ of the respondents reported no screening at all and 9\% did not have any clue regarding screening. Before surgery almost $58 \%$ of the orthopaedic surgeons were assessing the patients for COVID-19 mainly by signs and symptoms (51\%); only a small percentage (7\%) were taking swabs for PCR. The rest of the surgical community were not even assessing the patients for any signs and symptoms of COVID-19 before surgery. A total of $54 \%$ among them were not sure if they had operated on any 
Table I: Table showing survey questions that were asked from the participants regarding their practice

\begin{tabular}{|ll|}
\hline \# & Survey Questions regarding Practices in Outpatient, Emergency \& Operation theatres \\
\hline 1 & Has any SOPs for managing OPD been laid down by your department? \\
2 & Is your work setting still following the Pre-COVID 19 routine of outpatient clinic? \\
3 & If 'No' how it is reduced (check all that apply) \\
4 & Is your own personal private OPD practice has been changed? \\
5 & Which of the following are you using during patient care in OPD (check all that apply)? \\
6 & Did you observe any change in the patient turn out in OPD? \\
7 & Has your department started any telemedicine services during this pandemic? \\
8 & Has any SOPs for managing emergency services been laid down by your department? \\
9 & Emergency health care staff using which of the following during patient care (check all that apply)? \\
10 & On arrival to emergency services patients are screened for COVID 19 signs and symptoms like temperature, cough \\
& etc \\
11 & Has any SOPs for managing operation theatres been laid down by your department? \\
12 & What type of surgeries you are performing? \\
13 & Are you doing your private surgeries? \\
14 & Which of the following are you using during surgery (check all that apply)? \\
15 & Are you keeping your surgical team as minimal as possible? \\
16 & Are you screening your patients for COVID-19 before surgeries? \\
17 & Patients stay in hospital after surgical procedure has been minimized as much as possible \\
18 & Are you trying to keep your surgical time short? \\
19 & Have you attended/operated any COVID suspected/ positive patient? \\
20 & How stressed out are you with the current scenario \\
21 & Are you involved in teaching learning during this lockdown period? \\
22 & Are you involved in any research during this lockdown? \\
23 & Are you happy with your hospital response to COVID 19 pandemic? \\
&
\end{tabular}

Table II: Table showing respondents demographics and work setting details

\begin{tabular}{|c|c|c|c|c|c|c|c|c|c|c|c|c|c|}
\hline \multicolumn{5}{|c|}{ Age } & \multicolumn{2}{|c|}{$\begin{array}{l}\text { Completed } \\
\text { PG training }\end{array}$} & \multicolumn{3}{|c|}{ Practice } & \multicolumn{3}{|c|}{ Work setting } & \multirow[b]{2}{*}{ Multiple } \\
\hline $25-34$ & $35-44$ & $45-54$ & $55-64$ & $>65$ & Yes & No & Urban & Rural & Both & $\begin{array}{l}\text { Outpatient / } \\
\text { Private / } \\
\text { Specialty } \\
\text { clinic }\end{array}$ & $\begin{array}{l}\text { Private } \\
\text { Hospital }\end{array}$ & $\begin{array}{l}\text { Govt. } \\
\text { Hospital }\end{array}$ & \\
\hline $62 \%$ & $20 \%$ & $9 \%$ & $8 \%$ & $1 \%$ & $66 \%$ & $34 \%$ & $83 \%$ & $13 \%$ & $4 \%$ & $22 \%$ & $15 \%$ & $42 \%$ & $21 \%$ \\
\hline
\end{tabular}

Table III: Spearman rank correlation coefficient for comparison between safety practices in three areas of practices

\begin{tabular}{|lccc|}
\hline Safety Practices & OPD & ER & OT \\
\hline OPD & - & $0.38^{*}$ & $0.39^{*}$ \\
ER & $0.38^{*}$ & - & $0.21^{*}$ \\
\hline
\end{tabular}

Abbreviations; OPD: Outpatient Department, ER: Emergency, OT: Operation Theatre, * $\mathrm{P}<0.001$

COVID-19 patient or not, however, $10 \%$ confirmed to have operated COVID-19 patients.

In the current scenario of COVID-19 only $22 \%$ of the orthopaedic surgeons were doing both trauma and elective surgeries whereas $69 \%$ were doing only those trauma surgeries which could not be avoided and $9 \%$ were not doing any surgeries at all. Almost $64 \%$ had stopped doing surgery in private practice and $18 \%$ had minimised the number of surgeries performed. Nearly $70 \%$ of the orthopaedic surgeons were keeping their surgical time and team short and $78 \%$ had reduced the duration of patient's stay in hospital after surgery.
In response to general questions; $59 \%$ of the respondents mentioned that their departments had taken the steps for the provision of telemedicine services during this pandemic. Regarding involvement in teaching and learning during this partial lockdown period, 79\% confirmed their involvement mainly through webinars and zoom meetings and participation in research activity was only affirmed by $16 \%$ of individuals. In general $89 \%$ of the respondents indicated definite to mild stress and $63 \%$ showed dissatisfaction regarding the response of their hospitals to COVID-19.

There was a significant impact of age on the change in private practice $(p<0.001)$, almost $60 \%$ of the orthopaedic 


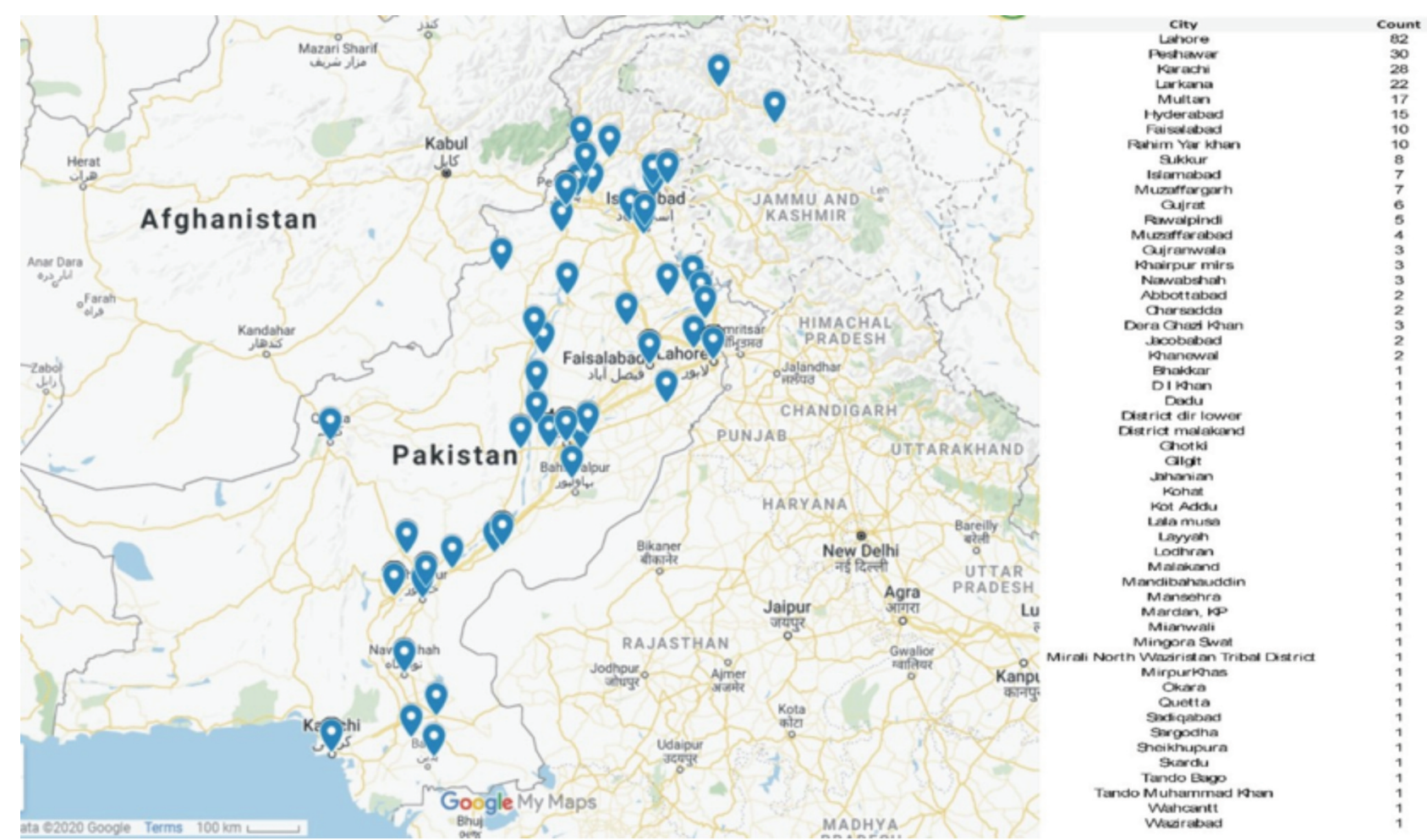

Fig. 1: Representative map drawn on Google map showing distribution of survey responses received from various cities of Pakistan. https://www.google.com/maps/d/u/0/edit?mid=1D5117_DtReSqUsvi4vlfTZXDmp5wcefK\&usp=sharing (accessed on 06th June 2020).

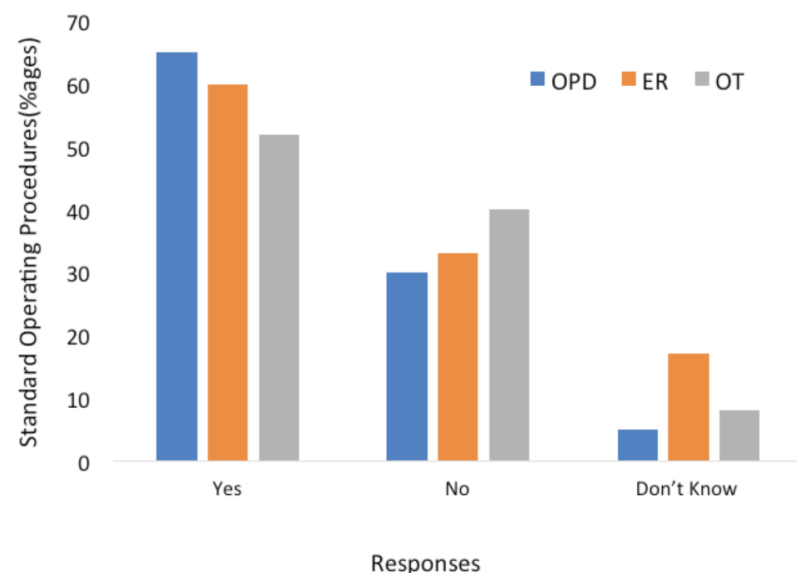

Fig. 2: Bar graph based on the responses showing outpatient departments (OPD), emergency (ER) and operation theatres (OT) with and without standard operating procedures.

consultants younger than 45 years had mentioned that they had stopped doing private surgeries compared to only $29 \%$ of the consultants above 45 years. Multiple logistic regression showed the strongest predictors of age $(\mathrm{p}=0.001)$ and completion of postgraduate training $(\mathrm{p}<0.001)$ in private practice surgery.

The age also has a significant effect on the use of protection gear during OPD services ( $\mathrm{p}=0.03$ ), the younger age group of orthopaedic surgeons were more careful and used better protection compared to the group above 45 years of age. The

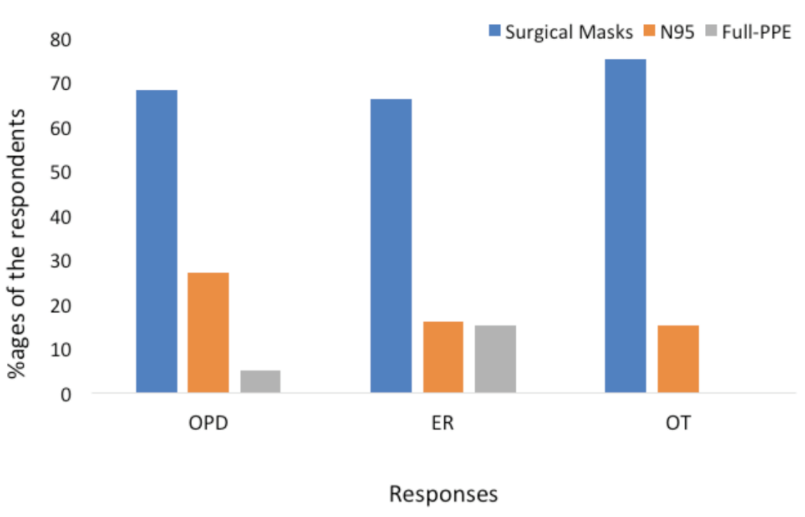

Fig. 3: Bar graph showing percentage of respondents using various safety measures like only surgical masks, N95 respirator and full personal protective equipment (PPE) in outpatient departments (OPD), emergency (ER) and operation theatres (OT).

orthopaedic surgeons working in rural setting were found to be more careful in practicing safety measures while working in OT $(p=0.003)$ using N95 mostly. Multiple logistic regression showed the strongest predictors of practice setting in rural area $(\mathrm{p}=0.01)$ in the use of protection measures.

With increasing age level of stress was significantly higher ( $p=0.01$ ), the orthopaedic surgeons above 45 years of age showed higher level of stress compared to the group below 45 years who were mostly mildly stressed. Multiple logistic regression analysis showed that the strongest predictor was of age $(\mathrm{p}=0.01)$. 


\section{DISCUSSION}

This study represents the first attempt to identify the OPD, ER and surgical practices among orthopaedic surgeons during the COVID-19 pandemic in Pakistan. Based on the responses it was noted that a huge number of hospitals and health care settings still lack SOP in their outpatient departments, emergency areas and especially in operating rooms. With this global pandemic, healthcare systems around the world are preparing themselves to take care of surgeries at more than maximum capacity for a number of months, therefore, proper guidelines and safety training in surgical services are crucial at this stage ${ }^{10,12-14}$. In these unique circumstances it is mandatory that hospitals and surgical units make comprehensive SOP which can be adapted by surgeons ${ }^{2}$.

Pakistan Orthopaedic Association (POA) has issued the "Standard Operating Procedure for COVID-19 Coronavirus Patients" "15 which should be strictly followed in hospitals and every orthopaedic surgeons should be made aware of, however, these guidelines need improvement and have failed to set the protocols to be followed in operating theatres. Many international surgical and orthopaedic departments and societies have already provided a frame work for the elective and urgent surgeries and careful planning has been introduced to minimise staff shortages related to uncontrolled viral spread ${ }^{13,16}$. Since Pakistan is far behind in the number of people tested for COVID-19 there is an estimate that there is huge number of asymptomatic patients in the local settings. A recent study done at an orthopaedic specialty hospital in New York City reported that 59\% COVID positive patients were asymptomatic among those screened prior to their planned orthopaedic surgical procedure $^{17}$. This shows the extreme likelihood of asymptomatic patients and the potential risk of transmission of the disease. Therefore, vigilant control measures are warranted at every step considering every patient as a possible source of infection.

Regarding the safety protocols, the responses of the orthopaedic surgeons were quite alarming in all the settings. It was revealed that most of the orthopaedics are using only surgical masks while attending patients, evidence suggests that surgical masks are not a reliable protection against COVID-19. The current survey further revealed that senior consultants above the age of 45 years have a more casual attitude towards self-safety measures and this raises the additional concerns with the possibility for them becoming infected and requiring self-isolation. This could potentially lead to a dangerous shortage of senior expertise within surgical teams and may increase the risk of cross infection to patients and others.

Healthcare professionals are quite vulnerable to the exposure of aerosols in the theatres, therefore, it is advisable for them to wear N95 masks and proper gear even during surgery ${ }^{4,10}$. It was quite interesting to find that in the rural health settings orthopaedic surgeons are more careful while operating as compared to urban settings. In the current scenario every patient should be treated as a potential COVID-19 positive case and it is logical that a surgical balaclava, gown and double gloves must be used as standard practice to minimise potential skin contact with the virus ${ }^{4}$. The guidelines published recently for orthopaedic departments on the basis of experience from Wuhan, People's Republic of China highly recommend that in OPD "orthopaedic surgeons should select appropriate personal protective equipment (PPE) based on Level-II protective standards, including a hair net, gloves, an isolation suit, a medical respirator (filtering face piece $[\mathrm{FFP}] / \mathrm{N}, \mathrm{R}$, or $\mathrm{P}$ ), eye protection (goggles and/or visor), and shoe covers" ${ }^{18}$. Recently a case report of a 64-year-old male patient who was tested positive for SARS-COV-2 and underwent explantation of a shoulder prosthesis due to a periprosthetic infection suggests that it is possible to prevent spread of infection among health care workers if strict control measures are taken. In this case all 66 health care workers remained safe and COVID-19 was not detected in any of them despite high-risk exposure during intubation, surgical treatment and general care ${ }^{19}$.

It was indicated through our survey that in most of the settings the screening of patient is usually through assessment of signs and symptoms leaving the gap of asymptomatic transmission and putting orthopaedic surgeons at high risk of infection. The recommendations of POA regarding the dress code and 3 ply surgical masks when not in COVID wards ${ }^{15}$ is quite perilous in these circumstances.

A large number in the orthopaedic community displayed higher level of stress and this was significantly higher in senior consultants above the age of 45 years. The stress may be due to loss of practice, financial burden and future uncertainty as most of the surgeons have either stopped their surgical practice and/or reduced it and were not happy with the steps taken by their administration in response to COVID. A disrupted life work balance with loss of financial support, emotional stress, fear of falling sick and health risks for the family could be factors for the mental stress in the orthopaedic fraternity ${ }^{20,21}$.

\section{CONCLUSION}

Our study strongly suggests that appropriate use of protective gear and infection control protocols are essential among the orthopaedic fraternity especially at this stage when Pakistan is facing a major outbreak of virus cases these days. The concerned societies and hospitals should take serious steps to outline and define the standard protocols at every step to minimise the transmission and cross infection.

\section{CONFLICT OF INTEREST}

The authors declare no potential conflict of interest. 


\section{REFERENCES}

1. Bornstein SS, Mire RD, Barrett ED, Moyer DV, Cooney TG. The Collision of COVID-19 and the U.S. Health System. Ann Intern Med. 2020: M20-1851. doi: 10.7326/M20-1851

2. Weiner JA, Swiatek PR, Johnson DJ, Louie PK, Harada GK, McCarthy MH, et al. Learning from the past: did experience with previous epidemics help mitigate the impact of COVID-19 among spine surgeons worldwide? Eur Spine J. 2020; 29: 1789-1805. doi: 10.1007/s00586-020-06477-6

3. Imielski B. The detrimental effect of COVID-19 on subspecialty medical education. Surgery. 2020; 168(2): 218-9. doi: 10.1016/j.surg.2020.05.012

4. Baldock TE, Bolam SM, Gao R, Zhu MF, Rosenfeldt MPJ, Young SW, et al. Infection prevention measures for orthopaedic departments during the COVID-2019 pandemic: a review of current evidence. Bone Jt Open. 2020; 1(4): 74-9. doi: 10.1302/2633-1462.14.BJO-2020-0018.R1

5. Athey AG, Cao L, Okazaki K, Zagra L, Castelli CC, Kendoff DO, et al. Survey of AAHKS International Members on the Impact of COVID-19 on Hip and Knee Arthroplasty Practices. J Arthroplasty. 2020; 35(7S): S89-94. doi: 10.1016/j.arth.2020.04.053

6. Khalid MOR, Zaheer R. The invisible victims - Impact of the pandemic on patients without COVID-19. J Pak Med Assoc. 2020; 70(Suppl 3)(5): S149-52. doi: 10.5455/JPMA.36

7. UN Office for the Coordination of Humanitarian Affairs. Pakistan: COVID-19 - Situation Report. 2020. https://reliefweb.int/report/pakistan/pakistan-covid-19-situation-report-1st-june-2020 (accessed on 01 June 2020)

8. Viswanath A, Monga P. Working through the COVID-19 outbreak: Rapid review and recommendations for MSK and allied heath personnel. J Clin Orthop Trauma. 2020; 11(3): 500-3. doi: 10.1016/j.jcot.2020.03.014

9. Al-Jabir A, Kerwan A, Nicola M, Alsafi Z, Khan M, Sohrabi C, et al. Impact of the coronavirus (COVID-19) pandemic on surgical practice - Part 2 (surgical prioritisation). Int J Surg. 2020; 79: 233-48. doi: 10.1016/j.ijsu.2020.05.002

10. Liu M, Cheng SZ, Xu KW, Yang Y, Zhu QT, Zhang H, et al. Use of personal protective equipment against coronavirus disease 2019 by healthcare professionals in Wuhan, China: cross sectional study. BMJ. 2020; 369. doi: 10.1136/bmj.m2195

11. Liang ZC, Wang W, Murphy D, Hui JHP. Novel Coronavirus and Orthopaedic Surgery: Early Experiences from Singapore. $J$ Bone Joint Surg Am. 2020; 102(9): 745-9. doi: 10.2106/JBJS.20.00236

12. Wang Y, Zeng L, Yao S, Zhu F, Liu C, Di Laura A, et al. Recommendations of protective measures for orthopedic surgeons during COVID-19 pandemic. Knee Surg Sports Traumatol Arthrosc. 2020; 28(7): 2027-35. doi: 10.1007/s00167-020-06092-4

13. Coccolini F, Perrone G, Chiarugi M, Di Marzo F, Ansaloni L, Scandroglio I, et al. Surgery in COVID-19 patients: operational directives. World J Emerg Surg. 2020; 15: 25. doi: 10.1186/s13017-020-00307-2

14. Chhabra HS, Bagaraia V, Keny S, Kalidindi KKV, Mallepally A, Dhillon MS, et al. COVID-19: Current Knowledge and Best Practices for Orthopaedic Surgeons. Indian J Orthop. 2020; 54(4): 411-25. doi: 10.1007/s43465-020-00135-1

15. Pakistan Association of Orthopedics. Recommend Policy and Standard Operating Procedure for COVID-19 Coronavirus Patients Screening. 2020. http://p-o-a.org/recommend-policy-and-standard-operating-procedure-for-covid-19-coronavirus-patientsscreening/ (accessed on 01 June 2020)

16. Ahmed S, Tan WLG, Chong YL. Surgical Response to COVID-19 Pandemic: A Singapore Perspective. J Am Coll Surg. 2020; 230(6): 1074-7. doi: 10.1016/j.jamcollsurg.2020.04.003

17. Gruskay JA, Dvorzhinskiy A, Konnaris MA, LeBrun DG, Ghahramani GC, Premkumar A, et al. Universal Testing for COVID19 in Essential Orthopaedic Surgery Reveals a High Percentage of Asymptomatic Infections. J Bone Joint Surg Am. 2020;102(16):1379-88. doi: 10.2106/JBJS.20.01053

18. Xiong Y, Chen L, Lin Z, Panayi AC, Mi B, Liu G. Orthopaedic Guidelines for the COVID-19 Post-Outbreak Period: Experience from Wuhan, People's Republic of China. J Bone Joint Surg Am. 2020; 102(15): e87. doi: 10.2106/JBJS.20.00773

19. Cheung KMC, Karppinen J, Chan D, Ho DWH, Song YQ, Sham P, et al. Prevalence and pattern of lumbar magnetic resonance imaging changes in a population study of one thousand forty-three individuals. Spine (Phila Pa 1976). 2009; 34(9): 934-40. doi: 10.1097/BRS.0b013e3181a01b3f 
20. Sahu D, Agrawal T, Rathod V, Bagaria V. Impact of COVID 19 lockdown on orthopaedic surgeons in India: A survey. J Clin Orthop Trauma. 2020; 11(Suppl 3): S283-90. doi: 10.1016/j.jcot.2020.05.007

21. Brooks SK, Webster RK, Smith LE, Woodland L, Wessely S, Greenberg N, et al. The psychological impact of quarantine and how to reduce it: rapid review of the evidence. Lancet. 2020; 395(10227): 912-20. doi: 10.1016/S0140-6736(20)30460-8 